\title{
New research in bladder cancer, ASCO-GU 2017
}

\author{
Peter C. Black, $M D^{\prime}$; Srikala Sridhar, $M D^{2}$ \\ 'University of British Columbia, Vancouver, BC; 2University of Toronto, Toronto, ON; Canada
}

(MDA) basal, and The Cancer Genome Atlas (TCGA) cluster III had the greatest improvement in overall survival (OS) after NAC compared to surgery alone, while those with UNC

\section{Intravesical rAd-IFN $\alpha /$ Syn3 for NMIBC refractory to BCG}

While intravesical bacillus Calmette-Guérin (BCG) is the recommended therapy for non-muscle-invasive bladder cancer (NMIBC), there is a subset of patients who are refractory to, or relapse on this therapy. A phase 2 trial presented at the American Society of Clinical Oncology 2017 Genitourinary Cancers Symposium (ASCO-GU) investigated a novel intravesical therapy, rAd-IFNa/Syn3, a replication-deficient, recombinant adenovirus gene transfer vector among 40 patients with high-grade (HG) BCG-unresponsive NMIBC. ${ }^{1}$ The subjects were randomized to receive the investigational therapy either at $1 \times 10^{11} \mathrm{vp} / \mathrm{mL}$ or $3 \times 10^{11} \mathrm{vp} / \mathrm{mL}$.

Fourteen of the 40 patients (35\%) were free of HG recurrence at 12 months (primary endpoint). There was no significant difference between the treatment arms; however, the median time to HG recurrence was longer for those in the higher-dose group (11.7 months) compared to the lowerdose group (3.5 months). The recurrence-free rate was 50\% among those patients with papillary-only $(\mathrm{Ta} / \mathrm{T} 1)$ disease (5/10 patients).

There were no Grade 4 or 5 treatment-emergent adverse events (TEAEs). There were two serious adverse events (SAEs) that resolved with medical treatment. The most common TEAEs were urinary (micturition urgency, $\mathrm{n}=15$; dysuria, $\mathrm{n}=11$; pollakuria, $\mathrm{n}=11$; nocturia, $\mathrm{n}=8$ ).

\section{Neoadjuvant chemotherapy in MIBC}

At ASCO-GU 2017, researchers presented their work investigating the impact of different molecular subtyping methods on response to neoadjuvant cisplatin-based chemotherapy (NAC) and developed a single sample model for subtyping. ${ }^{2}$ They reported that the benefit of NAC varies between molecular subtypes: individuals with tumours classified as University of North Carolina (UNC) basal, MD Anderson claudin-low tumours had the worst OS, irrespective of treatment regimen $(p=0.005)$. The survival rates with or without NAC are shown in Fig. 1. Given the impact of treatment on patients with basal tumours, the authors hypothesized that NAC could be prioritized for individuals with basal tumours, provided these results can be validated in further studies.

\section{Checkpoint inhibitors}

Research with checkpoint inhibitors continues to be a focus in GU cancers. At ASCO-GU 2017, there were several presentations of data from trials involving the PD-1 inhibitor pembrolizumab and the PD-L1 inhibitor atezolizumab.

For pembrolizumab, one of the key data sets presented come from a phase 2 study among 370 patients with cisplatinineligible advanced urothelial cancer (KEYNOTE-052). ${ }^{3}$ These patients, who had no previous systemic therapy for advanced disease, received intravenous pembrolizumab 200 mg every three weeks for 24 months or until disease progression.

The median duration of followup was five months (range $0.1-17)$. The overall objective response rate (ORR: complete or partial response, the primary endpoint) was $24 \%(95 \%$ confidence interval [CI] 20-29) (Table 1). Higher response rates were seen among those patients with greater PD-L1 expression. Among those with a combined positive score (number of PD-L1-positive cells divided by total tumour cells) of $\geq 10 \%$, the ORR was $48 \%$. Overall, $58 \%$ of patients had a reduction in tumour size from baseline in their target lesions. Among those who responded, 83\% were ongoing at the time of data cutoff. There were no new safety signals for pembrolizumab identified in this study.

A separate presentation provided health-related quality of life (HRQoL) data from another cohort treated with pembrolizumab. ${ }^{4}$ These patients were those in the KEYNOTE-045 study investigating pembrolizumab vs. investigator's choice chemotherapy among patients with previously treated advanced urothelial cancer $(n=520)$. In this study, pembrolizumab was associated with a prolongation of time to deteri- 


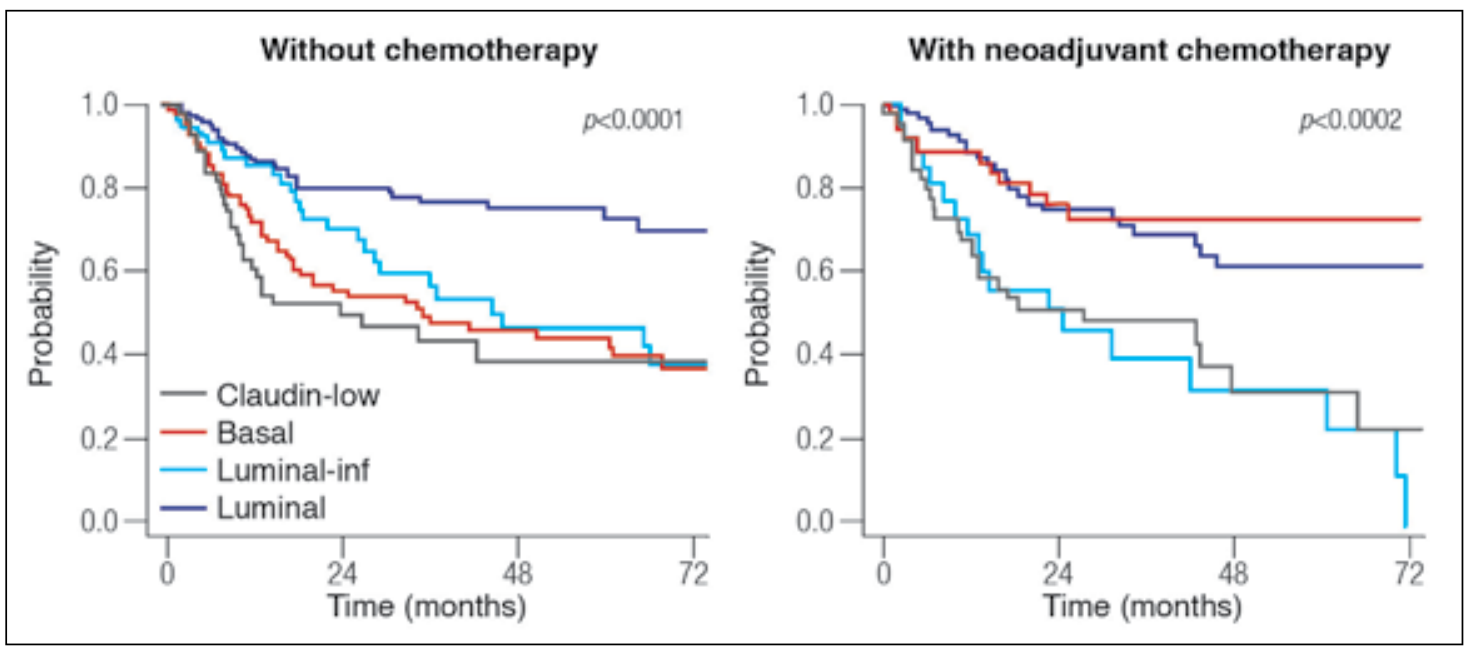

Fig. 1. Neoadjuvant chemotherapy for muscle-invasive bladder cancer: Overall survival by molecular subtype.

oration in HRQoL compared to chemotherapy (median 3.5 months vs. 2.2 months; $p=0.002$ ). Pembrolizumab was also found to be superior to chemotherapy across a spectrum of other HRQoL domains measured in the study (e.g., European Organization for Research and Treatment of Cancer core quality of life questionnaire [EORTC QLQ-C30], EuroQol five dimensions questionnaire [EuroQol EQ-5D]).

Researchers also presented some data with the PD-L1 inhibitor atezolizumab. This included a subgroup analysis of the phase 2 IMvigor210 study in patients previously treated with cisplatin-based therapy. ${ }^{5}$ The investigators subdivided this group by the number of lines of prior therapies received in the metastatic setting before study enrolment. They reported that clinical benefit with atezolizumab was observed regardless of the number of prior therapies for advanced disease, with no major differences in ORR, OS, or toxicity (Table 2).

There was also an update on long-term followup data from a phase 1 study with atezolizumab. ${ }^{6} \mathrm{~A}$ total of 95 patients with metastatic urothelial cancer were included in the expansion cohort and followed for a median of 29.2 months. The median duration of atezolizumab treatment was three months and five doses. The investigators reported that

Table 1. Confirmed objective response with pembrolizumab in cisplatin-ineligible patients (Phase 2 KEYNOTE-052)

\begin{tabular}{lcc} 
& \multicolumn{2}{c}{ Total population (n=370) } \\
\cline { 2 - 3 } & $\mathbf{n}$ & $\%(\mathbf{9 5 \%} \mathbf{C l})$ \\
\cline { 2 - 3 } Objective response rate & 89 & $24(20-29)$ \\
Complete response & 17 & $5(3-7)$ \\
Partial response & 72 & $19(16-24)$ \\
Stable disease & 84 & $23(19-27)$ \\
Progressive disease & 156 & $42(37-47)$ \\
No assessment & 31 & $8(6-12)$ \\
Not evaluable & 10 & $3(1-5)$ \\
\hline Cl: confidence interval. & & \\
\hline
\end{tabular}

there were no new safety signals with atezolizumab and that responses were durable ( $40 \%$ of responses were ongoing). Median OS was 10.1 months, with one- and two-year OS rates of $46 \%$ and $30 \%$, respectively.

\section{Review lectures in bladder cancer at ASCO-GU 2017}

One of the highlights of ASCO-GU 2017 was a review lecture presented by Dr. Elizabeth Plimack, discussing molecular predictors of response to NAC. ${ }^{7}$ She described genomic biomarkers of response, including the three-gene signature (ATM/RB1/FANCC) identified by her group and the ERCC2 mutation as described by Liu et al. She also showed how the likelihood of response to NAC is higher among those patients with greater number of genomic alterations. She presented the hypothesis behind these results, stating that defects in key DNA repair genes confer sensitivity to cisplatin chemotherapy. Dr. Plimack also showed how molecular subtypes may predict response to cisplatin-based NAC. Finally, she discussed the ongoing SWOG prospective clinical trial S1314, which is testing genomic signatures using the Co-expression Extrapolation (COXEN) model to predict response to neoadjuvant methotrexate, vinblastine, doxorubicin, and cisplatin (MVAC).

The value of genomics across the spectrum of bladder cancer was also discussed in a review lecture by Dr. David McConkey. ${ }^{8}$ Some additional points from his summary included that the immune landscape in luminal tumours (TCGA cluster I) may present challenges for the efficacy of immune checkpoint inhibitors, and that chemotherapy, Ad-IFN, and FGFR inhibitors may promote T-cell infiltration and increase sensitivity.

Finally, two speakers discussed their perspectives on trial design in NMIBC. Dr. Ashish Kamat ${ }^{9}$ presented four key myths about NMIBC from the urology perspective, which he said represent barriers to progress, and presented data 
Black et al.

\begin{tabular}{|c|c|c|c|c|c|c|}
\hline & \multicolumn{5}{|c|}{ Prior regimens for mUC } & \multirow{2}{*}{$\begin{array}{l}\text { All patients } \\
\qquad(n=310)\end{array}$} \\
\hline & $0(n=56)$ & $1(n=121)$ & $2(n=66)$ & $3(n=41)$ & $\geq 4(n=26)$ & \\
\hline Median OS $(95 \% \mathrm{Cl})$ & $\begin{array}{c}9.6 \mathrm{mos} \\
(5.9-15.6)\end{array}$ & $\begin{array}{c}9.0 \mathrm{mos} \\
(7.3-11.3)\end{array}$ & $\begin{array}{l}5.9 \text { mos } \\
(3.3-8.7)\end{array}$ & $\begin{array}{c}6.4 \mathrm{mos} \\
(3.8-10.2\end{array}$ & $\begin{array}{c}7.4 \mathrm{mos} \\
(4.6-11.2)\end{array}$ & $\begin{array}{l}7.9 \text { mos } \\
(6.7-9.3)\end{array}$ \\
\hline 12-month OS (95\% Cl) & $\begin{array}{c}45 \% \\
(32-58)\end{array}$ & $\begin{array}{c}38 \% \\
(29-47)\end{array}$ & $\begin{array}{c}34 \% \\
(23-46)\end{array}$ & $\begin{array}{c}33 \% \\
(18-47)\end{array}$ & $\begin{array}{c}28 \% \\
(10-46)\end{array}$ & $\begin{array}{c}37 \% \\
(31-42)\end{array}$ \\
\hline 18-month OS (95\% Cl) & $\begin{array}{c}34 \% \\
(21-46)\end{array}$ & $\begin{array}{c}34 \% \\
(21-46)\end{array}$ & $\begin{array}{c}28 \% \\
(17-39)\end{array}$ & $\begin{array}{c}28 \% \\
(14-42)\end{array}$ & $\begin{array}{c}20 \% \\
(4-36)\end{array}$ & $\begin{array}{c}27 \% \\
(22-32)\end{array}$ \\
\hline
\end{tabular}

$\mathrm{Cl}$ : confidence interval; mos: months; mUC: metastatic urothelial carcinoma; OS: overall survival.

to debunk each of these. The myths were: that NMIBC is a homogeneous disease; that the quality of transurethral resection (TUR) and cystoscopy is uniform; that BCG failure is well-defined; and that urologists follow guidelines. Addressing each of these barriers, he argued, would lead to more meaningful research in NMIBC.

In the same session, Dr. Noah Hahn ${ }^{10}$ presented and debunked four myths from the oncology perspective. These myths were: that small tissue samples preclude genomically enriched trial designs; that systemic therapy does not effectively reach the urothelium; that phenotypic heterogeneity and infinite targets prevent novel efficient trial designs; and that intravesical therapies are sufficient to improve outcomes.

\section{References}

1. Boorijan S, Shore N, Canter D, et al. Intravesical rad-IFN $\alpha /$ Syn3 for patients with high-grade, bacillus Calmette-Guérin (BCG) refractory or relapsed non-muscle-invasive bladder cancer: A phase 2 randomized study. Presented at ASCO-GU 2017; abstract \#279.
2. Seiler R, Winters B, Douglas J, et al. Muscle-invasive bladder cancer: Molecular subtypes and response to neoadjuvant chemotherapy. Presented at ASCO-GU 2017; abstract \#281.

3. Balar AV, Castellano D, O'Donnell PH, et al. Pembrolizumab as first-line therapy in cisplatin-ineligible advanced urothelial cancer: Results from the total KEYNOTE-052 study population. Presented at ASCO-GU 2017; abstract \#284.

4. Vaughn DJ, Bellmunt J, de Wit R, et al. Health-related quality of life (HRQL) in the KEYNOTE-045 study of pembrolizumab vs. investigator-choice chemotherapy for previously treated advanced urothelial cancer. Presented at ASCO-GU 2017; abstract \#282.

5. Perez-Gracia JL, Yohann L, Rosenberg JE, et al. Atezolizumab (atezo) in platinum-treated locally advanced or metastatic urothelial carcinoma (mUC): Outcomes by prior therapy. Presented at ASCO-GU 2017; abstract \#323.

6. Petrylak D, Powles T, Bellmunt J, et al. Atezolizumab (atezo) in patients with metastatic urothelial carcinoma (mUC): A two-year clinical update from a phase la study. Presented at ASCO-GU 2017; abstract \#290.

7. Plimack E. Molecular predictors of response to neoadjuvant chemotherapy. Presented at ASCO-GU 2017.

8. McConkey D. Emerging impact of genomics on the clinical management of bladder cancer. Presented at ASCO-GU 2017.

9. Kamat AM. Perspectives on trial design in NMIBC: Barriers to progress. Presented at ASCO-GU 2017.

10. Hahn 0. Perspectives on trial design in NMIBC: Oncology perspective. Presented at ASCO-GU 2017. 